\title{
Refractory status epilepticus responding to lidocaine: A case report
}

\author{
Gökhan Özer \\ Department of Neurology, Sanko University School of Medicine, Gaziantep, Turkey
}

\begin{abstract}
Status epilepticus is a life-threatening condition, which is associated with a high mortality and morbidity when left untreated. It is defined as a continuous seizure lasting more than $30 \mathrm{~min}$ or successive seizures with no recovery between any of them. Mortality of status epilepticus depends on the duration and type of seizure, concomitant diseases and age of the patient. Management of status epilepticus includes ensuring adequate airway, stabilization of circulation and use of antiepileptic drugs. Benzodiazepines, phenobarbital, phenytoin, fosphenytoin, general anesthesia and lidocaine are used for the treatment of status epilepticus. Here, we discuss a patient with refractory status epilepticus who achieved a very good response and full recovery only with lidocaine infusion among all forms of therapy given.
\end{abstract}

Eur Res J 2018;4(3):254-257

Keywords: status epilepticus, lidocaine, antiepileptic drugs

\section{Introduction}

Two or more successive epileptic seizures that fail to respond to antiepileptic drug therapy should be considered drug-resistant and a distinct treatment strategy should be followed in such patients. This presentation of epilepsy usually involves respiratory and cardiovascular problems and associated systemic complications. Status epilepticus has a high mortality when left untreated. Status epilepticus mortality is higher in the elderly and lower in childhood. In many cases, systemic and metabolic derangements related to status epilepticus cause neuronal damage and cellular impairment. Refractory status epilepticus is a form of epilepsy that fails to respond to therapy and involves continued seizure activity as demonstrated by clinical and electroencephalographic findings. Several antiepileptic agents are used for its treatment at predefined dose ranges and protocols $[1,2]$. In this paper, we will discuss a case of refractory status epilepticus in a case with no previously diagnosed epilepsy who reported having ten generalized tonicclonic seizures successively on the same day at her first presentation and responded only to lidocaine despite administration of full range of antiepileptic drug therapies.

\section{Case Presentation}

A 16-year-old female, right-handed student was 


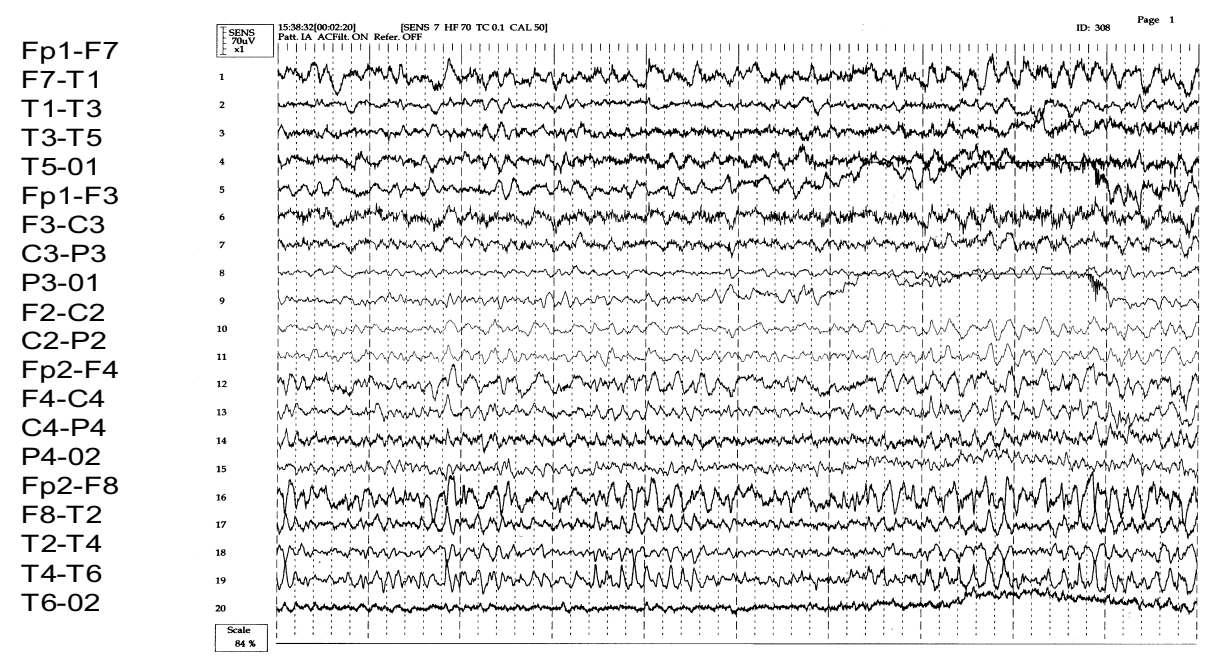

Figure 1. Ictal activity originating from the right frontotemporal area.

brought by her family to our emergency room after experiencing ten generalized tonic-clonic seizures successively on the same day with loss of consciousness. She had no history of seizures and suffered a complex partial seizure that developed a week ago involving gazing at a fixed point, not responding when questions were directed and pulling on her clothes obsessively 2-3 times a day. Prior to admission to emergency room, she had a total of six generalized tonic-clonic seizures in the last 3 days. Neither personal nor family history indicated remarkable risk factors or characteristics. On neurological examination, she was unconscious, nonresponsive to verbal and painful stimuli. Brainstem reflexes were present with bilaterally extensor plantar skin reflex and no facial asymmetry.

During her emergency room stay, she developed sudden and rapid onset of eye deviation, head deviation to the right with persistent generalized tonicclonic seizures. An emergency computed tomography scan of the brain did not show acute pathology. While her vital findings were stabilizing, diazepam was administered intermittently at a dose of $30 \mathrm{mg}$ /day by intravenous route. The patient was transferred to the neurology intensive care unit (ICU) and phenytoin infusion was given at a dose of $30 \mathrm{mg} / \mathrm{kg}$. During that period, she was unconscious with persistent complex partial and generalized tonic-clonic seizures, eye deviation, and hand automatism; therefore, she was intubated and midazolam infusion $(0.4 \mathrm{mg} / \mathrm{kg} / \mathrm{h})$ was initiated. At 12 hours after her admission, her clinical seizure activity decreased, and vital findings were stabilized and a lumbar puncture was performed. Cerebrospinal fluid examination and cranial magnetic resonance imaging showed normal findings.

An electroencephalogram showed continuous seizure activity originating from the right frontotemporal area spreading to the left and right hemispheres (Figures 1, 2a and 2b). Thus, phenytoin and midazolam infusion were maintained on the

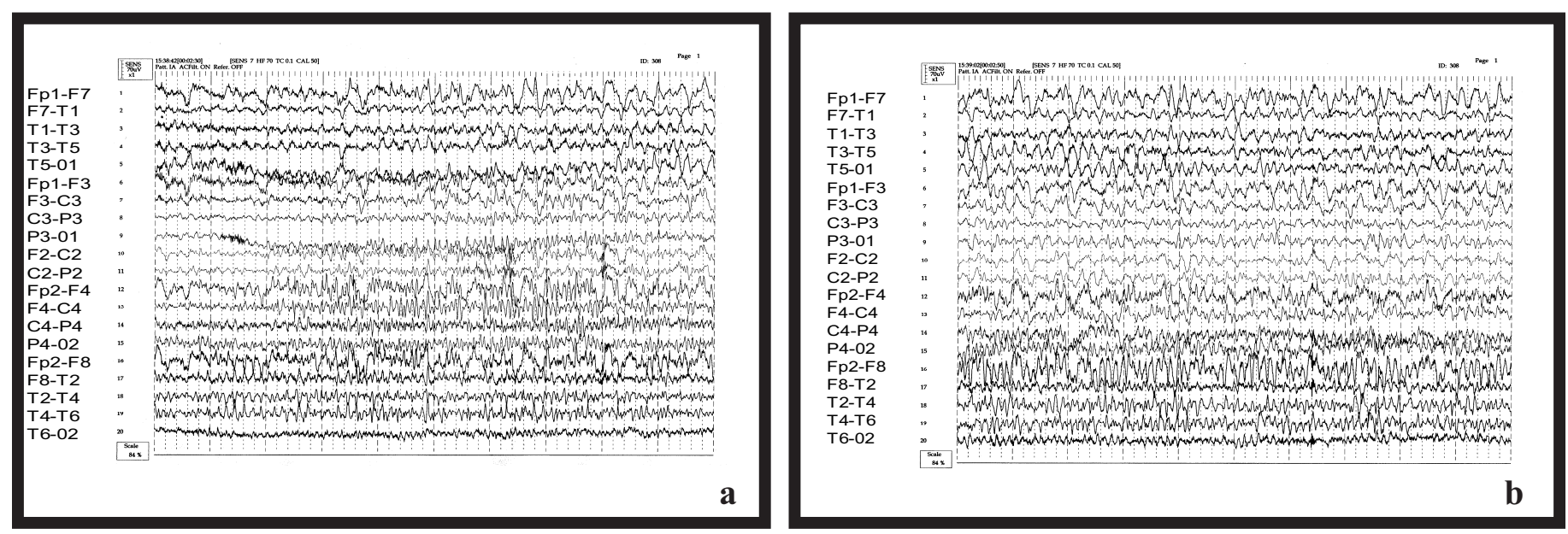

Figure 2. Spreading of the ictal activity originating from the right frontotemporal area to the left hemisphere (a). Spreading of the ictal activity originating from the right frontotemporal area to the right hemisphere (b). 
second day of her admission. A follow-up electroencephalogram on day 3 showed persistent seizure activity; therefore, carbamazepine was added to the treatment, thiopental infusion $(5 \mathrm{mg} / \mathrm{kg} / \mathrm{h})$ was initiated and midazolam infusion discontinued by tapering. Since clinical and electrophysiological seizures were resolved at that time, attempts were made to reduce thiopental infusions on two occasions, but seizure activity recurred first electrophysiologically and then clinically. Thus, lidocaine infusion $(3 \mathrm{mg} / \mathrm{kg} / \mathrm{h})$ was started on day 8 and thiopental infusion was tapered and then stopped altogether. On the third day of lidocaine infusion, full seizure control was achieved both electrophysiologically and clinically. She gradually gained consciousness and was maintained on phenytoin $3 \times 125 \mathrm{mg}$ and carbamazepine $1200 \mathrm{mg} /$ day thereafter.

During her hospitalization, she was put on and weaned from mechanical ventilation four times and in addition to her manifestations, pneumonia, gastrointestinal bleeding, elevation of liver function tests and thrombocytopenia developed. Topiramate was added to her treatment with gradual discontinuation of phenytoin.

During follow-up, the patient became fully conscious and alert and was transferred from the ICU to the neurology ward on day 15 . The patient is currently on maintenance therapy with topiramate 200 $\mathrm{mg}$ /day and carbamazepine $1200 \mathrm{mg}$ /day with no seizures for 2.5 years.

\section{Discussion}

Epileptic patients may enter the phase of status epilepticus at some point during the course of their disease with an incidence of 1.3-1.6\%. The most common cause of status epilepticus is the withdrawal of the antiepileptic drug. Status epilepticus is associated with a high rate of mortality which ranges from 8 to $32 \%$. This rate may vary depending on the underlying etiology and concomitant conditions. Permanent neurological sequelae can occur in patients with prolonged status epilepticus. Cerebral damage related to status epilepticus may also develop entirely independently of systemic complications. This kind of independent neuronal damage is believed to develop as a result of increased metabolic demand through calcium-dependent excitotoxicity, glial metabotropic glutamate receptors and excessive neuronal activity
[1-3]. Recent arguments suggest that certain surgical epilepsy interventions (such as focal resection or multiple subpial transection) may be needed for the treatment of refractory status epilepticus in carefully selected cases who are nonresponsive to repeated courses of medical therapy. Prognosis is often poor in refractory status epilepticus but the observation that many patients achieved full neurological recovery over the years suggests that it might be wrong to stop treatment prematurely [4].

A crucial step in the management of status epilepticus is "suspecting, scanning and sorting" which involves suspecting the disease in an early stage, performing neuroradiological imaging studies needed for the diagnosis and obtaining an electroencephalogram. Treatment should begin with stabilization of the vital findings, followed by correction of any electrolyte imbalance and resolution of fever. Since the risk of secondary infection is high, treatment with an appropriate antibiotic should be initiated and a lumbar puncture performed for differential diagnosis when deemed necessary. For specific treatment, antiepileptic therapies should be used by careful patient monitoring in an intensive care setting since these medications are toxic and may lead to respiratory depression. Benzodiazepines are firstline therapy. Diazepam, lorazepam and midazolam are the most commonly used agents. They have a rapid onset of action and penetrate readily into the cerebral tissue due to high fat solubility. Their major side effects include respiratory depression. Broad-spectrum antiepileptic drugs such as phenytoin and fosphenytoin are used in the second-line treatment as loading and maintenance doses in status epilepticus. Phenobarbital may be given to patients who do not benefit from benzodiazepines or phenytoin with vigilance for respiratory depression [5].

Parenteral valproate has been used since 1997. It can be used for rapid seizure control and in patients who cannot be orally fed. Recent studies comparing the efficacy of parenteral phenytoin and parenteral valproate in the treatment of refractory status epilepticus showed no statistically significant difference. It was suggested that valproate may be a good alternative to phenytoin particularly in patients with cardiopulmonary disease [6].

Additionally, intravenous levetiracetam may be used safely for the treatment of benzodiazepinerefractory status epilepticus in selected cases, especially in patients with liver failure, elevated liver enzymes or cardiac arrhythmias [7]. For treatment of 
status epilepticus refractory to phenytoin and benzodiazepines, lidocaine, rectal valproate, magnesium sulfate, rectal benzodiazepine and rectal chloral hydrate may be used [8]. General anesthesia may be performed in case of failure to achieve response to these therapies.

Lidocaine was first used in 1955 by Bernhard, Boem and Hojebag. Since then, it has been used in patients non-responsive to diazepam, phenytoin, phenobarbital or general anesthetic agents. Lidocaine is a sodium channel blocker. There are both individual case reports and case series published on refractory status epilepticus responding to intravenous lidocaine. Since lidocaine does not cause respiratory depression, it was suggested that it might be good therapeutic option particularly in patients with a chronic lung disease or no response to diazepam. Generally, it is recommended that administration of lidocaine should be continued until epileptic seizure resolves. Some studies have reported both lack of efficacy and harmful effects of lidocaine in many patients. Therefore, administered lidocaine dose should not exceed 2-3 $\mathrm{mg} / \mathrm{kg}$ of body weight [9].

Generalized status epilepticus has an average mortality rate of $20 \%$ (range, $3-35 \%$ ). Anorexia is the most common cause of mortality $(60 \%)$. The currently presented case is particularly interesting since the patient responded very well only to lidocaine despite administration of several lines of therapy and no occurrence of neurological deficits although she was put to and weaned from mechanical ventilation many times and treated in the ICU with a clinical picture of status epilepticus for two weeks.

\section{Conclusions}

Refractory status epilepticus is a difficult-to-treat condition which demands patience and a step-wise management approach. Throughout the course of treatment, seizures occurring in the patient should be clearly identified both clinically and by electroencephalogram and the choice of drug should be based on the age, metabolic condition of the patient and underlying cause of the disease.

\section{Informed consent}

Written informed consent was obtained from the patient and patient's family for the publication of this case report.

\section{Conflict of interest}

The authors declared that there are no potential conflicts of interest with respect to the research, authorship, and/or publication of this article.

\section{Authors contribution}

Author were involved in the study conception and design. Author provided input to the discussion, interpreted the findings, assisted in writing of the draft manuscript, reviewed the manuscript for intellectual content, and read and approved the final manuscript.

\section{References}

[1] Canas N, Delgado H, Silva V, Pinto AR, Sousa S, Simões R, et al. The electroclinical spectrum, etiologies, treatment and outcome of nonconvulsive status epilepticus in the elderly. Epilepsy Behav 2017;79:53-7.

[2] Brophy GM, Bell R, Claassen J, Alldredge B, Bleck TP, Glauser T, et al. Guidelines for the evaluation and management of status epilepticus. Neurocrit Care 2012;17:3-23.

[3] Towne AR, Pellock JM, Ko D, DeLorenzo RJ. Determinants of mortality in status epilepticus. Epilepsia 1994;35:27-34.

[4] DeLorenzo RJ, Ko D, Towne AR, Garnett LK, Boggs JG, Waterhouse EJ, et al. Prediction of outcome in status epilepticus. Epilepsia 1997;38(suppl 8):2105.

[5] Riviello JJ Jr, Claassen J, LaRoche SM, Sperling MR, Alldredge B, Bleck TP, et al. Treatment of status epilepticus: an international survey of experts. Neurocrit Care 2013;18:193-200.

[6] Lempl Y, Eshel Y, Gilad R, Sarova-Pinchas I. Chloral hydrate in intractable status epilepticus. Ann Emerg Med 1990;19:674-6.

[7] Knake S, Gruener J, Hattemer K, Klein KM, Bauer S, Oertel WH, et al Intravenous levetiracetam in the treatment of benzodiazepine-refractory status epilepticus. J Neurol Neurosurg Psychiatry 2008;79:588-9.

[8] Agarwal P, Kumar N, Chandra R, Gupta G, Antony AR, Garg N. Randomized study of intravenous valproate and phenytoin in status epilepticus. Seizure 2007; 16:527-32.

[9] Mori K, Ito H, Toda Y, Hashimoto T, Miyazaki M, Saijo T, et al. Successful management of intractable epilepsy with lidocaine tapes and continuous subcutaneous lidocaine infusion. Epilepsia 2004;45:1287-90. 